\title{
Drei Jahre Fraunhofer Anwendungszentrum "Entsorgungs- und Verkehrslogistik" an der Technischen Fachhochschule Wildau
}

\author{
Prof. Dr-Ing. habil. Bernd Hentschel
}

\section{Einleitung}

Im Rahmen einer bereits vor der Wendezeit bestehenden wissenschaftlich - technischen Zusammenarbeit zwischen einem Berliner Werkzeugmaschinenkombinat und dem Fraunhofer Institut fuir Materialfluß und Logistik entstand der Gedanke, mit der Wiedervereinigung in Berlin durch Ausgründung eines kleinen mittelständischen Logistikdienstleisters eine Außenstelle für Produktionslogistik des Dortmunder Fraunhofer Institutes zu errichten.

Als Logistikdienstleister empfahl sich die Berliner Firma „Projektlogistik GmbH - Planung, Entwicklung und Realisierung von Logistiksystemen“. So wurde mit Wirkung vom 01.01.1991 auf Vorschlag des Institutsleiters, Herrn Prof. Dr.-Ing. Dr.h.c.mult.Dr.-Ing.E.h. R. Jünemann, am Sitz des Berliner Logistikdienstleisters die erste Außenstelle „Produktionslogistik“ des Fraunhofer Institutes fuir Materialfluß und Logistik in den Neuen Bundesländern aufgebaut. Es erfolgte eine mehrjährige erfolgreiche Projektzusammenarbeit.

Mit der Berufung des dortigen Geschäftsfuihrers an die Technische Fachhochschule Wildau für das Lehrgebiet Produktionslogistik wurde in gemeinsamer Arbeit zwischen dem Grïndungsrektor, Herrn Prof. Dr.-Ing. W. Arlt, und dem Fraunhoferinstitutsleiter der Vorschlag erörtert, erstmalig für Deutschland ein neues Modell zu realisieren. Das Pilotvorhaben lautete:

Aufbau eines Fraunhofer-Anwendungszentrums mit Arbeitssitz an einer deutschen Technischen Fachhochschule realisieren und den vollen Arbeitsbetrieb für eine Applikationsprojektarbeit sichern.

Nach erfolgreicher Verhandlung zwischen der Fraunhofer Gesellschaft in München und dem Land Brandenburg wurde per 01.01.1995 formal die Bildung des Fraunhofer Anwendungszentrums ,Entsorgungs- und Verkehrslogistik" mit Sitz an der Technischen Fachhochschule Wildau beschlossen sowie ein Kooperationsvertrag abgeschlossen; exakte Arbeitsaufnahme war dann der 01.

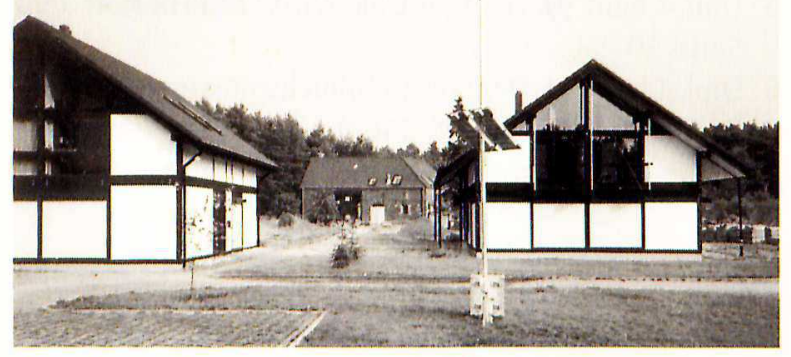

Bild 1: Fraunhofer Anwendungszentrum am Standort Prieros
März 1995 am Standort Wildau. Durch die vorhandene Raumsituation am Standort Wildau wurde es zum unmittelbaren Betreiben des Anwenderzentrums notwendig, an einem zweiten Standort in Prieros eine zusätzliche Investition von ca. 4,7 Mio DM privat zu investieren, um ein repräsentatives Logistikzentrum für das Land Brandenburg vorweisen zu können (Bild 1).

In diesem Zusammenhang muß gesagt werden, daß gerade in dieser schwierigen Situation die Unterstuitzung durch das Land Brandenburg nicht ausreichend war und somit eine große Chance der Wissenschaftslandschaft vernachlässigt wurde. Mit der Fraunhofer Gesellschaft hatte sich eine der renommiertesten Forschungseinrichtungen in Deutschland in Brandenburg etabliert und dies verdeutlichen allein Fakten zur Gesellschaft:

- derzeit 46 Institute

- ca. 8000 Beschäftigte

- 1,1 Mrd. DM Haushalt, davon 0,9 Mrd. DM Vertragsforschung

Einzig und allein das Engagement des Gründungsrektors der TFH Wildau und des Institutsleiters des Fraunhofer Institutes für Materialfluß und Logistik führte zu einer spïrbaren Unterstuitzung und fruchtbringenden Zusammenarbeit und somit zu einem erfolgreichen Anlauf des Anwenderzentrums. Dafür sei an dieser Stelle dem heutigen Präsidenten, Herrn Prof. Dr.-Ing. W. Arlt, und dem Institutsleiter des Fraunhofer Institutes, Herrn Prof. Dr.Ing. Dr. h.c.mult. Dr.-Ing. E.h. R. Jünemann, Dank gesagt.

\section{Arbeitsaufgaben und Ziele des Fraunhofer Anwendungszentrums}

Die Ziele der Fraunhofer Institute und somit des Fraunhofer Anwendungszentrums ergeben sich aus den Maximen des Namensgebers Joseph von Fraunhofer und ihrer Ergänzung aus heutiger Sicht. Sie lauten u. a.:

- grundsätzliche Vereinigung wissenschaftlicher Arbeit

- mit unternehmerischem Handeln und sofortige Umsetzung der Ergebnisse in den unternehmerischen Ablauf;

- strikte Realisierung anwendungsorientierter Forschungs- und Projektarbeit für Unternehmen und Kommunen;

- unternehmensbezogene Vertragsforschung finanziert zu $80 \%$ die Fraunhofer-Einrichtung und Zuordnung einer erfolgsabhängigen Zuschußfinanzierung aus Bund und Ländern.

Inhaltlich sowie beratungsseitig wurde der Rahmen global wie folgt definiert (Bild 2 und Bild 3 ). 


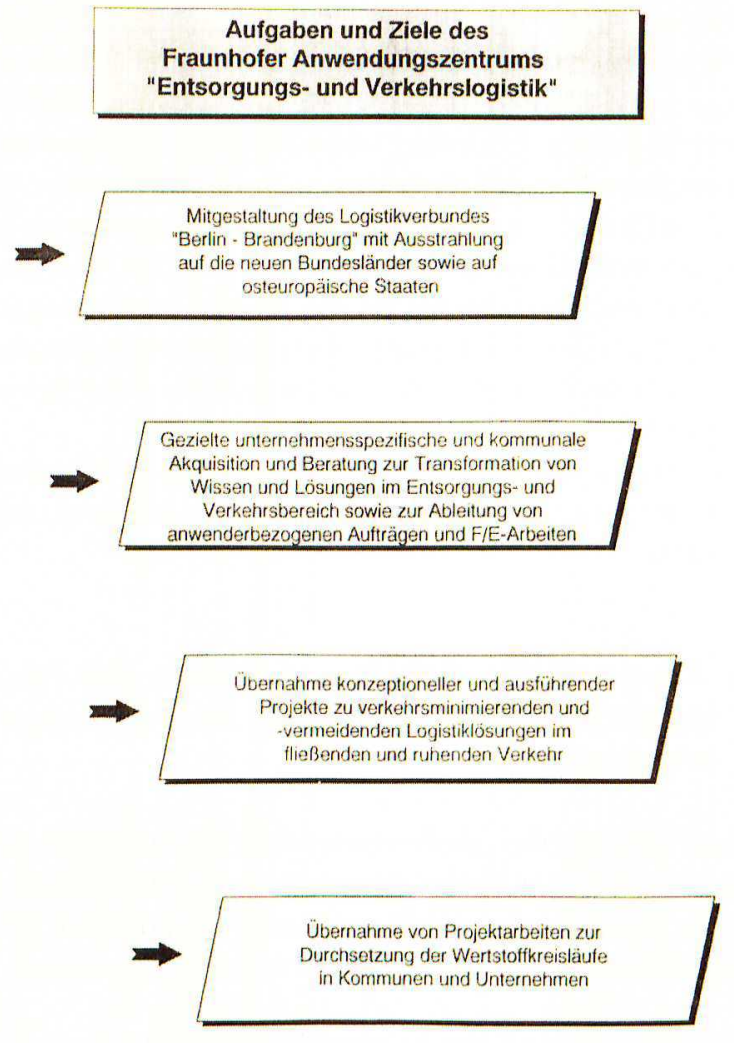

Bild 2: Aufgaben und Ziele des Fraunhofer Anwendungszentrums

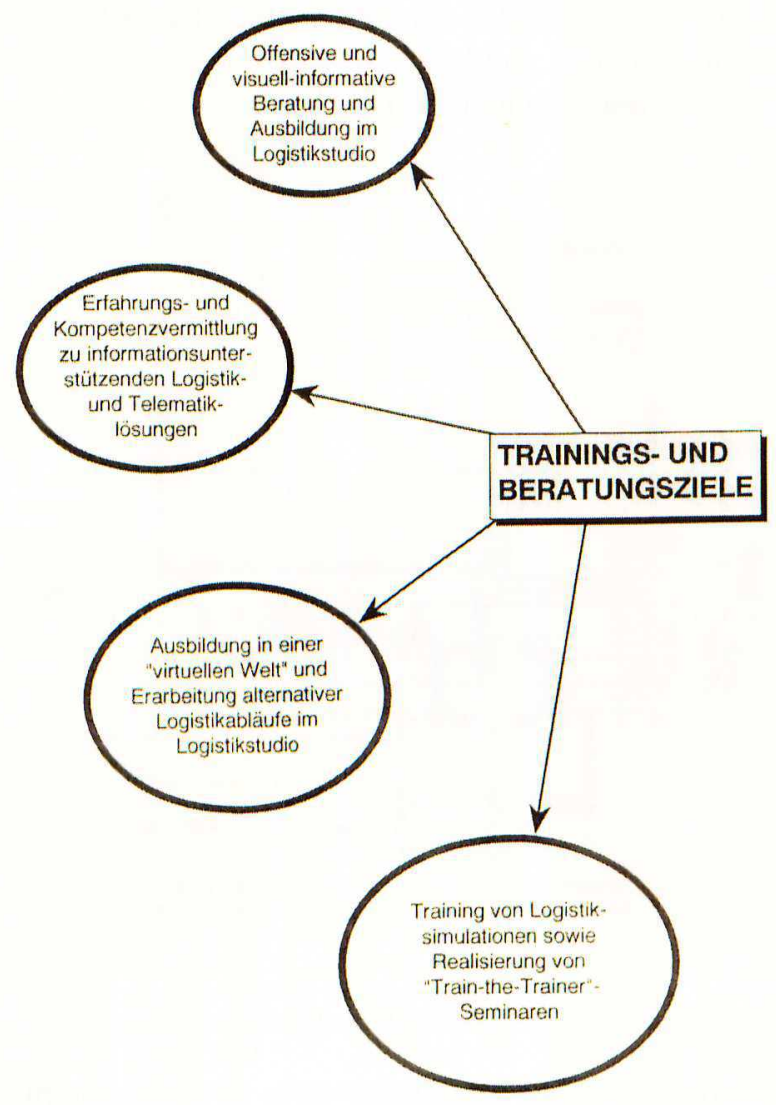

Bild 3: Trainings- und Beratungsziele
Mit diesen Prämissen ist klar, daß ein außerordentlich hoher Projektdruck und Betriebshaushaltszwang das Betreiben einer derartigen Einrichtung sichert. So wurde für den ersten dreijährigen Betreiberabschnitt mit grundsätzlichem Akquisitionspotential in den Neuen Bundesländern als auch ausgewählten osteuropäischen Staaten, insbesondere Polen, folgender Betriebshaushalt geplant (Auswahl):

\begin{tabular}{lrrr} 
& 1995 & 1996 & 1997 \\
\hline feste Mitarbeiter & 4 & 5 & 7 \\
Wirtschaftserträge & 500 TDM & 950 TDM & 1300 TDM \\
Erstausstattung & 1200 TDM & 900 TDM & 300 TDM \\
lfd. Investitionen & - TDM & 150 TDM & 200 TDM
\end{tabular}

Die regionalen und iiberregionalen Akquisitionsbereiche wurden mit den folgenden Schwerpunkten gesehen. Bild 4 stellt die angedachte Akquisitionsverteilung dar.

\section{Regionale / Überregionale Akquisitionsbereiche}

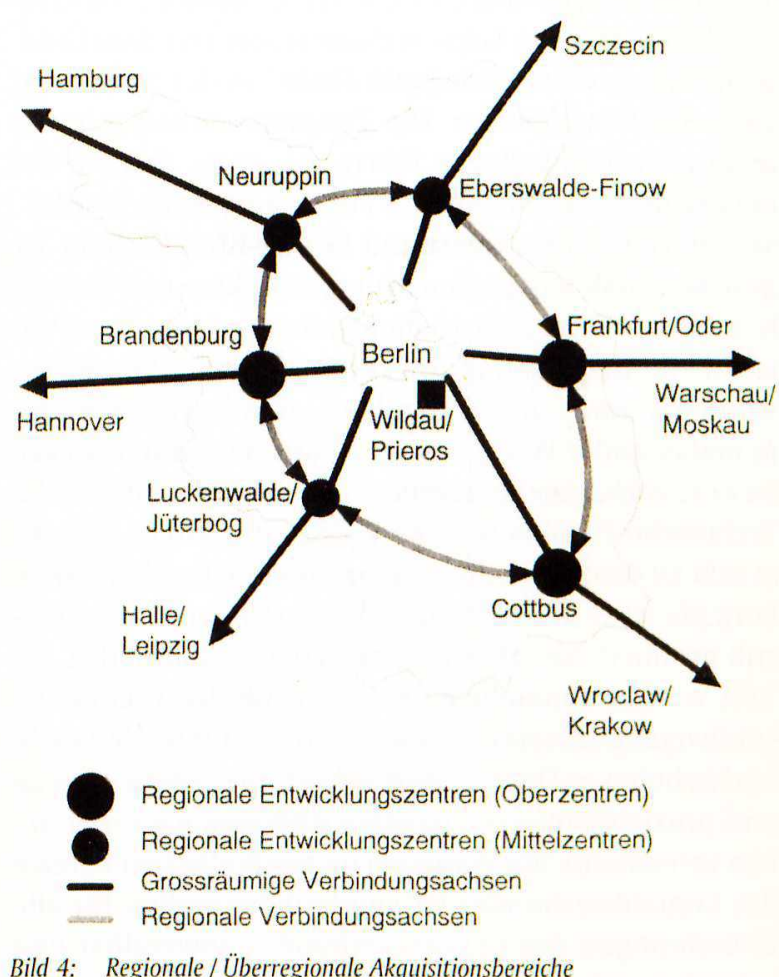

Alle Investitionen wurden zielgerichtet in innovative Hard- und Softwarelösungen sowie zum Betreiben von Logistik- und Beratungsstudios an der TFH Wildau und am Zweitstandort Prieros eingesetzt. So wurde am Standort Wildau das Logistikstudio „Produktionslogistik" hard- und softwareseitig errichtet (Finanzierung erfolgte über die TFH Wildau) und weiterhin auf Mietbasis im Sondermaschinenbau ein Versuchsfeld für neue innovative Produkte angemietet. Die Logistikstudios „Entsorgungslogistik“ und „Verkehrslogistik“ in Prieros sowie die dort ansässigen Mitarbeiter wurden mit den neuesten hard- und softwareseitigen Logistikkomponenten ausgestattet (Finanzierung uiber die Fraunhofer Gesellschaft), um für diese zukunftsträchtigen Projektfelder erfolgreich akquirieren zu können. 


\section{LOGISTIK - VERBUND BERLIN - BRANDENBURG}

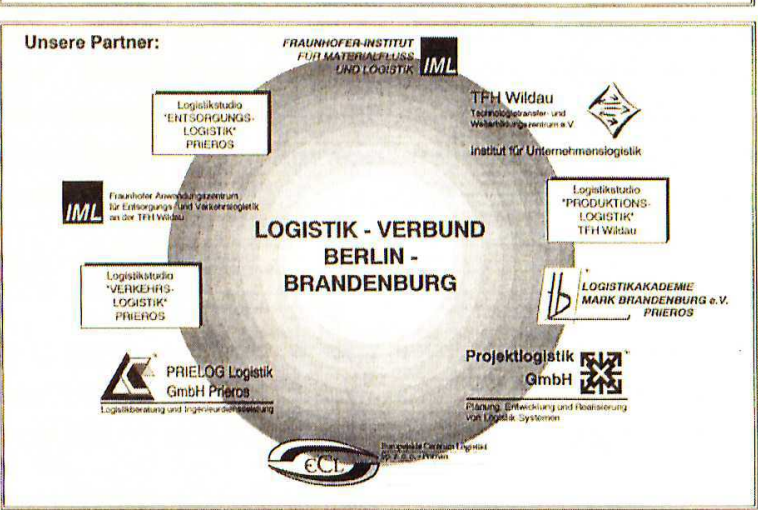

Bild 5: Logistikverbund Berlin-Brandenburg

Mit diesem Haushalt und in Zusammenarbeit mit dem Mutterinstitut in Dortmund sowie der Technischen Fachhochschule Wildau wurde dazu ein strategisches Konzept als Logistikverbund Berlin-Brandenburg erarbeitet und umgesetzt (Bild 5).

Ersichtlich ist die Mischung aus reinen Logistikdienstleistern im angewandten F/E-Bereich sowie die Integration kommerzieller Logistikdienstleister mit dem Gründungsmitglied Projektlogistik GmbH an der Spitze und anderen Dienstleistern. Die Zusammenarbeit mit den polnischen Fachkollegen fuihrte u.a. dazu, daß ein Gemeinschaftsunternehmen in Posen gegriindet wurde. Mit diesem Logistikverbund Berlin-Brandenburg ist gesichert, daß mit großen Synergieeffekten und kleinen leistungsstarken Logistikdienstleistern komplexe Projekte von der Planung bis zur Realsierung bearbeitet werden können und Technologie- und Logistiktransfer in umfassender Weise betrieben werden kann. Insofern ist eine wirksame Verbundlösung vorhanden, in der die Technische Fachhochschule Wildau mit ihrem Potential sich zu einem Kristallisationspunkt im Land Brandenburg als auch für die Neuen Bundesländer in der Logistik profiliert hat. Dies äußert sich auch darin, daß die TFH Wildau nunmehr einen eigenständigen Logistikstudiengang anbietet, und somit zu den führenden Fachhochschulen in Deutschland gehört, die eine wirksame und praxisorientierte Logistikausbildung garantieren. Das vereinheitlichte Vorgehen im methodischen Bereich des Logistikverbundes ist empfohlenermaßen für alle Einrichtungen des Logistikverbundes anwendbar und orientiert sich an den im Bild 6 ausgewiesenen Prämissen. Ziel ist dabei immer eine neutrale Vorgehenswei-

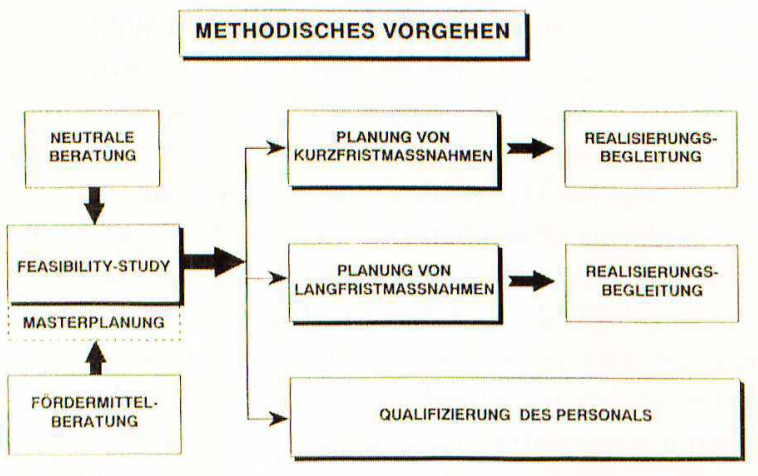

Bild 6: Methodisches Vorgehen im Logistikverbund se ohne Bindung an bestimmte Produzenten von Ausrüstungen, Hard- und Software zu garantieren. Oberster Grundsatz bleibt stets die Kundenorientierung. Selbstverständlich gehört auch die kostenlose Fördermittelberatung zu den Aufgaben. Gerade in den Neuen Bundesländern ist diese Komponente unverzichtbarer Bestandteil jedweder Logistikarbeit. Für das Fraunhofer Anwendungszentrum ist das Projekt erst beendet, wenn auch die Realisierungsbegleitung abgeschlossen und erforderlichenfalls die parallele Ausbildung beendet wurde.

Mit diesen wesentlichen Voraussetzungen konnten mehrere Projekte erfolgreich bearbeitet und realisiert werden.

\section{Ausgewählte Arbeitsergebnisse der Pro- jektarbeit des Fraunhofer Anwendungs- zentrums}

Nachfolgend sollen stellvertretend für die ausgeführten Projekte des Fraunhofer Anwendungszentrums 5 praktische Beispiele dargestellt und somit die erreichte Leistungsfähigkeit der Mannschaft verdeutlicht werden.

\subsection{Entwicklung einer automatisierten Demontage- zelle für Altfernsehgeräte}

In Zusammenarbeit mit dem Mutterinstitut und dem Logistikdienstleister PRIELOG Logistik GmbH Prieros wurde der erste Prototyp einer automatisierten Demontagezelle fuir Fernsehgeräte entwickelt und im Versuchsfeld am Standort des Sondermaschinenbaues Wildau $\mathrm{GmbH}$,in Stahl und Eisen“ realisiert. Bild 7 stellt das Layout dieser Demontagezelle dar.

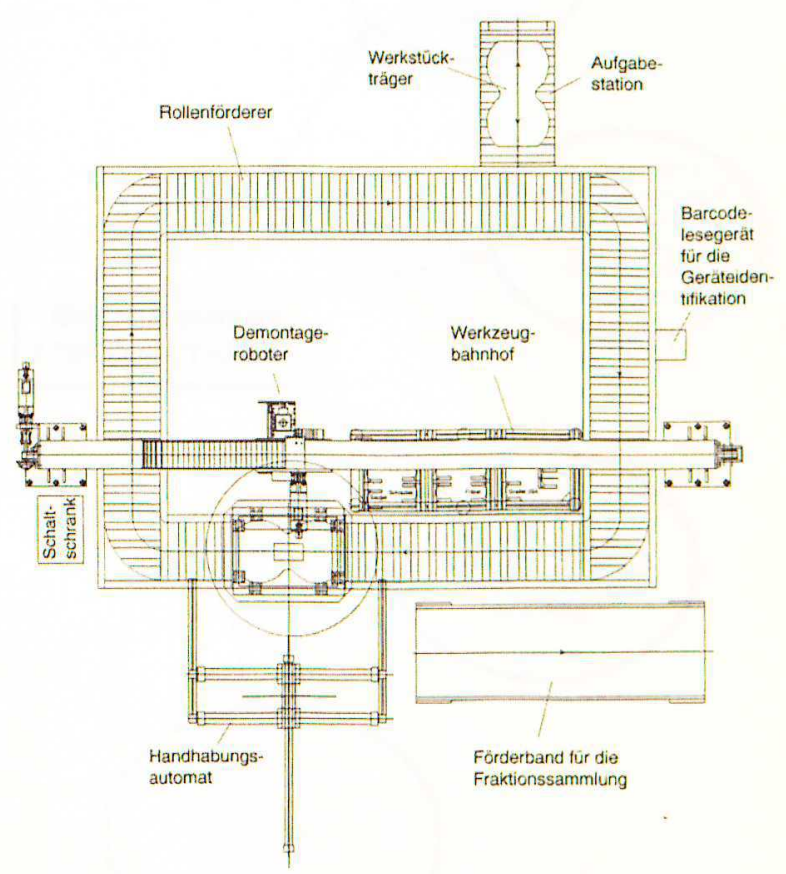

Bild 7: Konzept einer automatisierten Demontagezelle für Fernsehgeräte

Mit diesem Prototyp wurde erstmalig eine Zellenlösung für die Entsorgungslogistik realisiert und für die Praxis ein Modellfall aufgebaut, der sich gut für die Akquisition und Auftragseinwerbung eignet. Diese Lösung stellt 
in ihrer Grundkonzeption eine außerordentlich innovative Lösung dar und wird bei weiterer inhaltlicher Vervollkommnung mit Sicherheit zu einer neuen Produktvariante (vielleicht auch für ein Unternehmen im Land Brandenburg) fuihren. Anfragen namhafter Produzenten liegen vor und es besteht sicher die Möglichkeit, bei Einführung der Rücknahmeverpflichtung für Hersteller mit mehreren Entwicklungen zu rechnen.

Das Prinzip der Demontagezelle ist recht einfach. Über eine Aufgabestation werden die Fernsehgeräte einer Materialflußlösung zugeführt und an einer Geräteidentifikation vorbeigeführt. Derzeit läuft das System mit einer Barcodeidentifikation und so wird gesichert, daß das Gerät erkannt und uiber eine im Rechner hinterlegte Demontagetechnologie von einem Linienportalroboter demontiert werden kann. Die gegenwärtige Demontage orientiert sich an groben Fraktionierungsstufen und fuihrt in der Weiterführung zu spezifizierten Demontagelösungen für feinere Fraktionierungsstufen. Derzeit führt das Fraunhofer Anwendungszentrum für ein Wildauer Unternehmen die konzeptionelle Arbeit zur Entwicklung von Entstiickungsautomaten durch.

Die weitere Arbeit ist in Verbindung mit den Logistikdienstleistern des Logistikverbundes Berlin-Brandenburg darauf gerichtet, einen Demontagebaukasten für Demontagen der Braunen und Weißen Ware zu konzipieren. Hierzu liegen konkrete Aufträge mittelständischer Unternehmen aus Sachsen-Anhalt sowie Sachsen vor und berechtigen zu der Annahme, ein echtes innovatives und zugleich vermarktungsfähiges Produkt den Unternehmen zur Verfuigung zu stellen.

Damit wird ganz im Sinne der Fraunhofer Philosophie gearbeitet und die entwicklungsseitige Arbeit mit der unternehmerischen Komponente verbunden.

Strategisches Ziel ist es, den Standort Wildau als ein Zentrum fuir Demontagesysteme zu profilieren und mit den ansässigen Unternehmen durch derartige Entwicklungen neue Arbeitsplätze zu schaffen.

Hierzu dient auch die neue Initiative der Technischen Fachhochschule Wildau in Verbindung mit dem Fraunhofer Anwendungszentrum eine „Recyclinginitiative Brandenburg" zu entwickeln und durchgehende Logistikketten mit neuen Logistikdienstleistungen und Logistikprodukten fuir Brandenburger Unternehmen zu gestalten.

\subsection{Entwicklung einer Füllstandsfernabfrage für Wertstoffcontainer zur Optimierung der Fahr- zeugeinsatzplanung}

Diese spezifische Entsorgungslogistiklösung wurde in unserem Auftrag vom Mutterinstitut in Dortmund entwickelt.

Ausgangspunkt ist dabei die Erkenntnis, daß Wertstoffcontainer in städtischen Gebieten oder Ballungsräumen des öfteren uibervoll sind und es so zu größeren Schmutzplätzen kommt. Das Prinzip ist denkbar einfach. über einen im Container installierten Sensor kann ein Füllgrad definiert werden. Dieser wird so bemessen, daß er vielleicht $80 \%$ Füllhöhe ausmacht. Wird dieser definierte Füllstand erreicht, so aktiviert der installierte Sensor über eine Datenfernuibertragungseinheit den Zentralrechner des fuir die Entsorgung zuständigen Entsor- gungsunternehmens. Mit dieser Information versehen, wird dann tag-oder stundenkonkret die entsprechende Entsorgung eingeleitet und bei Bedarf die durch das Entsorgungsfahrzeug optimiert zu fahrende Route vorgegeben. Bild 8 verdeutlicht den prinzipiellen Aufbau.

\section{Entsorgungslogistik}

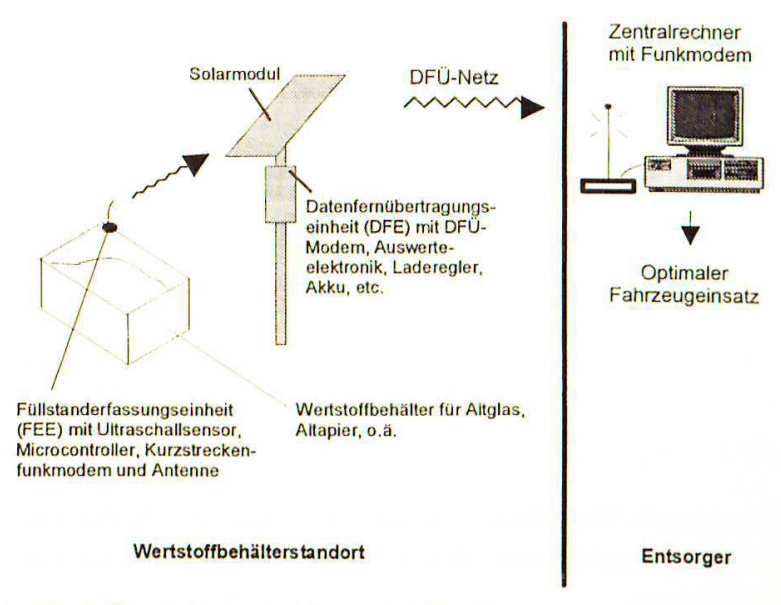

Bild 8: Fïllstandsfernabfrage für Wertstoffcontainer

Diese Lösung ist ebenfalls als Demonstrationsobjekt an der TFH Wildau sowie am Standort Prieros des Fraunhofer Anwendungszentrums installiert.

Selbstverständlich sind wesentlich andere Einsatzfälle denkbar und an dieser Problematik wird derzeit gearbeitet. Auch diese Lösung paßt sich in die Fraunhofer Philosophie ein und stellt in erster Linie eine sofort umsetzbare Innovation dar.

\subsection{Entwicklung einer Fertigungszelle für die auto- matisierte Produktion ïberlanger Befestigungs- elemente in der Rostocker Maschinenbau und Technologie GmbH \& Co. KG}

Dieses Projekt resultiert aus der langjährigen Zusammenarbeit mit dem ehemaligen Dieselmotorenwerk Rostock GmbH (heute: Dieselmotorenwerk Vulkan GmbH). Als reines produktionslogistisches Projekt ist hier das Problem der Fertigung uiberlanger Befestigungselemente (Zylinderkopfschrauben in der Länge von $500 \mathrm{~mm}$ bis $3000 \mathrm{~mm}$ ) im automatisierten Zyklus zu lösen. Die zu gestaltende Logistikkette fängt bei der Bereitstellung von Rohlingen an und umfaßt den robotergestuitzten Materialtransport von diesem Bereitstellplatz über die mechanische Drehbearbeitung mit allen Zu- und Abführprozessen, den erforderlichen Weitertransport zur automatischen Gewindewalzmaschine mit allen $\mathrm{Zu}$ - und Abfuihrprozessen, der automatischen Prüfung der Fertigteile und eindeutigen Kennzeichnung sowie die nachfolgenden Konservierungs- und Verpackungsprozesse. Diese Aufgabe ist in ihrer Komplexität außerordentlich schwer und verlangt höchsten ingenieurbezogenen und logistischen Einsatz. Infolge des außerordentlichen Risikos fuir den Auftraggeber wird diese Auftragserteilung von der Arbeitsgemeinschaft industrieller Forschungsvereinigungen e.V. (AiF) gefördert. 


\section{FFS - RMT (Variante 3)}

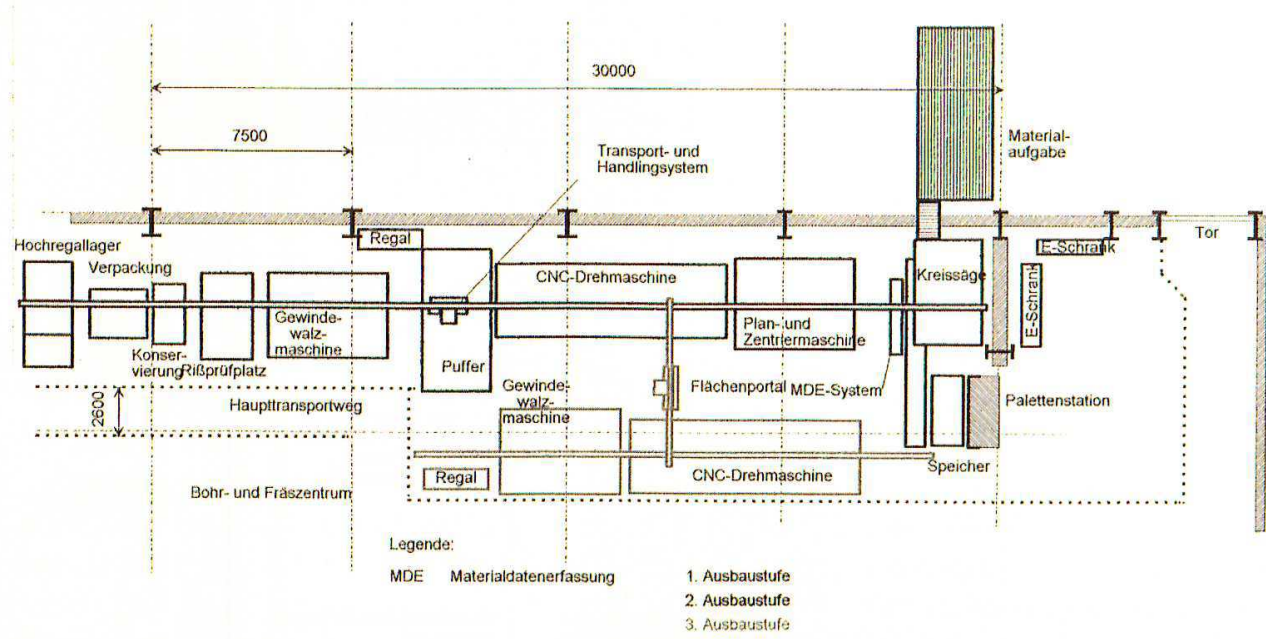

Bild 9: Konzept einer Fertigungszelle zur automatisierten Fertigung überlanger Befestigungselemente

chert werden können. Auch Telelearning und Telewartungskonzepte gehören dazu.

Die Akquisitionspotentiale erstrecken sich von der Erarbeitung einer videokonferenzorientierten Lösung für den globalen Austausch von konstruktiven Details innerhalb des Entwicklungsprozesses eines Automobilherstellers bis hin zur Erarbeitung von Telewartungskonzepten eines mittelständischen Unternehmens im Land Brandenburg.

Parallel wird diese videokonferenzorien-

Aus einem Stufenkonzept aufbauender Lösungsvarianten soll eine Variante durch Bild 9 die Komplexität verdeutlichen.

Diese Lösung selber ist bestens geeignet, in Teilen oder als Ganzes getreu dem Fraunhoferschen Gedanken vermarktet zu werden. Ein gemeinsames Vermarktungskonzept mit dem Auftraggeber Rostocker Maschinenbau und Technologie GmbH \& Co. KG ist erarbeitet. Der Abschluß der Realisierung und die Inbetriebnahme ist für 6/98 fixiert.

\subsection{Einsatz von Telelearningsystemen zur Verkehrs- vermeidung}

Im Rahmen der Verkehrslogistik ist die inhaltliche Ausrichtung auf die Konzipierung und Projektrealisierung verkehrsvermeidender Lösungen ausgerichtet. Insbesondere durch Einsatz von Informations- und Kommunikationslösungen wird der verkehrsvermeidende Ansatz in folgender Form gesehen: Nicht Kilotonnen transportieren, sondern Kilobytes auf Reise bringen!

Nur in dieser Form ist der zukünftige Anstieg im Individual- und Güterverkehr zu bewältigen; das bedeutet, Überlegungen anzustellen, inwieweit überflüssiger Verkehr vermieden werden kann und dennoch für die Unternehmen und Kommunen die erforderlichen Abstimmungen und Handlungsweisen gesi- tierte Technik auch zur Verbesserung der Vorlesungen an der Technischen Fachhochschule Wildau im Fach „Grundlagen der Logistik“ zum Einsatz gebracht.

Auf der Ersten Deutsch-Polnischen Logistikkonferenz an der TFH Wildau 1996 wurde bereits demonstriert, welche ausgezeichneten Möglichkeiten diese neue Kommunikationsform zur Qualifizierung der Ausbildung für unsere Studenten bietet. Bild 10 stellt den prinzipiellen Aufbau einer Telelearninglösung zwischen der Technischen Fachhochschule Wildau und unserer Partnerhochschule in Posen dar. Im Zeitraum 1997/98 wird gemeinsam mit unserem polnischen Partner für das Fachgebiet „Produktionslogistik“ eine Pilotlösung installiert und in den Vorlesungsbetrieb integriert.

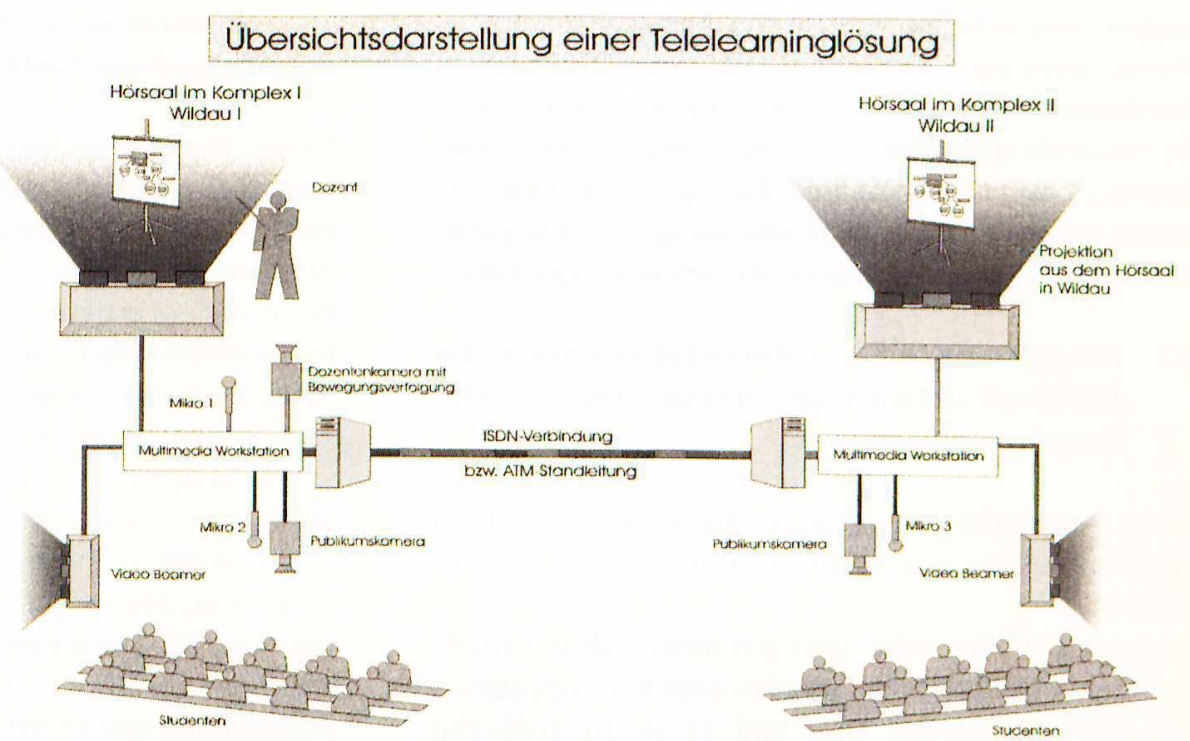

\section{DEUTSCH - POLNISCHE LOGISTIKKONFERENZ TECHNISCHE FACHHOCHSCHULE WILDAU 1996 \\ Prof. Dr.-Ing. habil. B. Hentschel}

Bild 10: Übersichtsdarstellung einer Telelearninglösung zwischen der TFH Wildau und der TU Posen (Darstellung zur Ersten Deutsch-Polnischen Logistikkonferenz) 
3.5. Durchführung der Deutsch-Polnischen Logistikkonferenz als ständige Einrichtung zum Logistikund Technologietransfer unter Führung der TFH Wildau und des Fraunhofer Anwendungszentrums

Nicht nur die konkrete unternehmensbezogene Projektarbeit steht im Vordergrund, sondern auch der permanente Wissens- und Logistik- sowie Technologietransfer nimmt eine bedeutsame Rolle in der gemeinschaftlichen Arbeit der Technischen Fachhochschule Wildau und dem Fraunhofer Anwendungszentrum ein.

In gemeinsamer Initiative wurde dazu das Forum der Deutsch-Polnischen Logistikkonferenz in wechselseitiger Veranstaltung in Polen und Deutschland geschaffen.

Die erste Veranstaltung dazu wurde unter der Schirmherrschaft des Ministerpräsidenten des Landes Brandenburg, Herrn Dr. M. Stolpe, sehr erfolgreich mit 159 Teilnehmern am 06./07.März 1996 an der Technischen Fachhochschule Wildau realisiert.

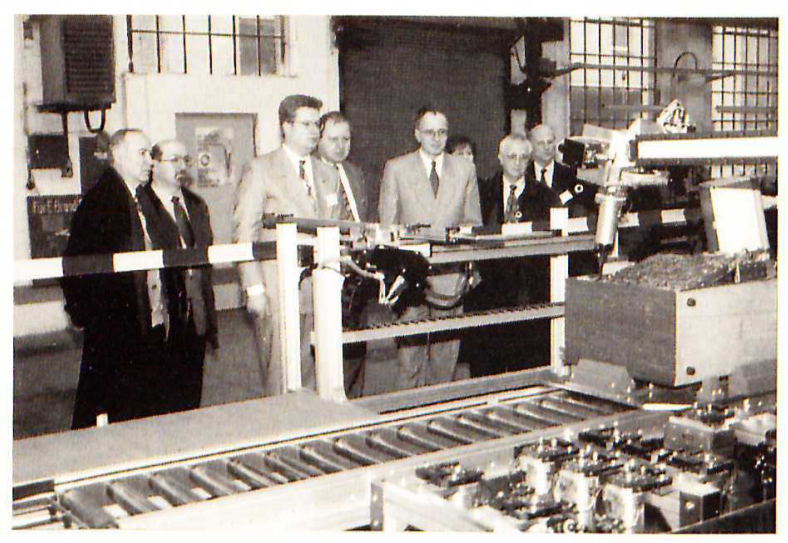

Bild 11: Minister Reiche an der Demontagezelle

In den einzelnen Sektionen der Produktions-, Entsorgungs- und Verkehrslogistik sowie für die Logistikausbildung wurden Vorträge gehalten und intensive inhaltliche Anregungen in den Workshops gegeben. Das Motto bestand fuir diese Konferenz in der erstmaligen Zusammenkunft deutscher und polnischer Spezialisten im Logistikfachbereich bzw. im Abgrenzen gemeinsam bestehender Interessen und gemeinsamer Projektarbeiten.

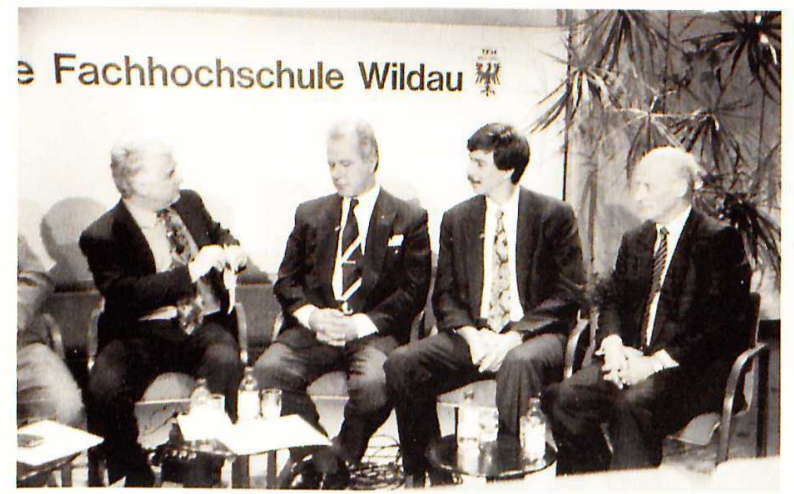

Bild 12: Fernsehdiskussion zur Ersten Deutsch-Polnischen Logistikkonferenz (v.l. Moderator Dr. Büchel, Herr Fiege von Fiege Logistik GmbH, Dr. Badke vom Sondermaschinenbau Wildau GmbH, Prof. Hentschel von der TFH Wildaul)
Während dieser Konferenz wurde die Inbetriebnahme der bereits angesprochenen Demontagezelle im Beisein des Ministers für Wissenschaft, Forschung und Kultur des Landes Brandenburg, Herrn Steffen Reiche, vorgenommen. Die Bilder 11 und 12 zeigen dazu einen Ausschnitt aus der Vorstellung der Demontagezelle sowie aus der anschließenden Fernsehdiskussion im regionalen Fernsehsender Puls-TV mit dem Chefredakteur Dr. Buichel.

Für 1997 ist die nächste Konferenz, diesmal als „Zweite Polnisch-Deutsche Logistikkonferenz" in Posen, unter dem Generalthema „Logistikinnovationen für Produkte und Dienstleistungen in der Wirtschaft" am 16./ 17.04.97 geplant.

Diese Konferenz steht diesmal unter der Schirmherrschaft des Bundesministers fuir Verkehr, Herrn M. Wissmann, und des polnischen Ministers für Verkehr und Seewirtschaft, Herrn B. Liberadzki. Beide Minister begriißen diese Konferenz als willkommenes Forum der Logistik und des weiteren Kennenlernens deutscher und polnischer Logistikspezialisten.

Somit wird auch durch diese permanente Transferarbeit der Fraunhofer Gedanke lebendig gehalten und ein wirksamer Beitrag zwischen Ländern erbracht.

Das Deckblatt der Konferenz - hier in Bild 13 ausgewiesen - wird sich nach Meinung aller veranstaltenden Parteien als nicht mehr wegzudenkendes Element in den deutsch-polnischen Logistikbeziehungen und die Veranstaltung als permanente Einrichtung einprägen.

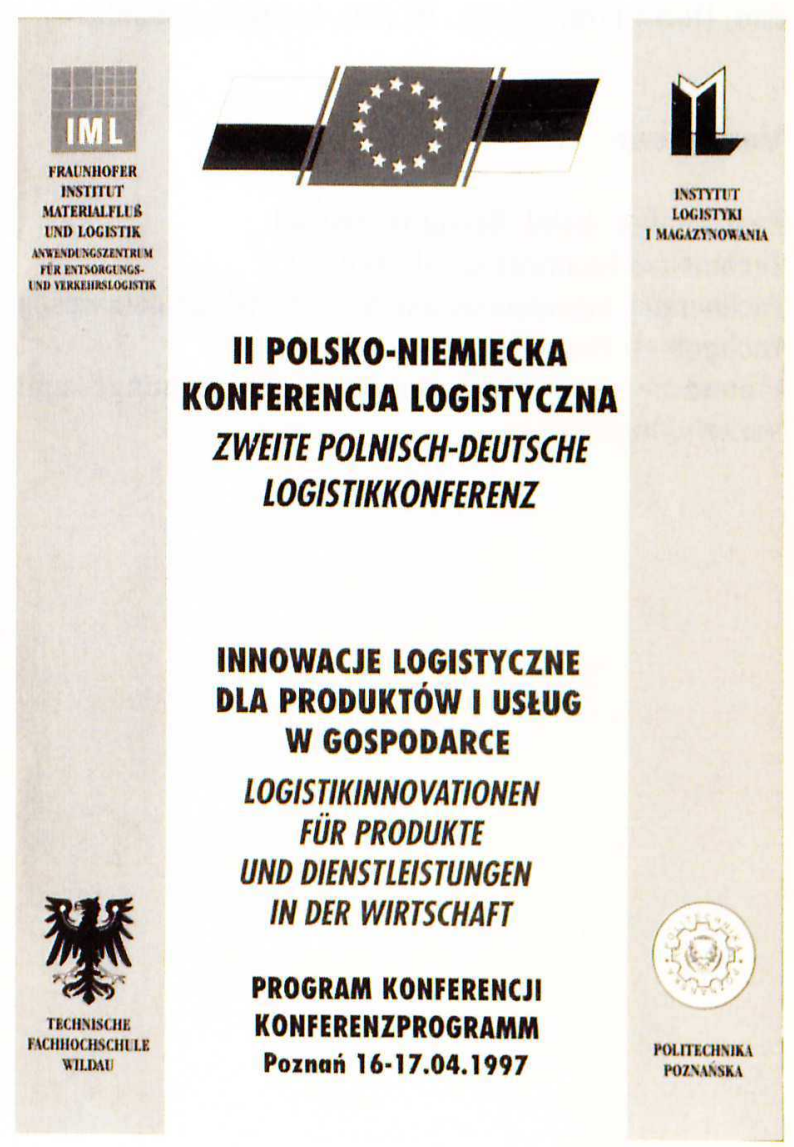

Bild 13: Einladung für die 2. Polnisch-Deutsche Logistikkonferen 1997 in Posen 


\section{Schlußbemerkungen}

Mit der Grïndung des Fraunhofer Anwendungszentrums „Entsorgungs- und Verkehrslogistik“ an der Technischen Fachhochschule Wildau wurde erstmalig in Deutschland ein Pilotprojekt derart gestartet, daß die Fraunhofer Gesellschaft ein Anwendungszentrum an eine Fachhochschule vergeben hat.

Nach zweijähriger Aufbauphase und Projektarbeit kann eindeutig festgehalten werden, daß sich dieser Schritt aus Sicht der Technischen Fachhochschule Wildau und aus Sicht des Fraunhofer Institutes für Materialfluß und Logistik als richtig und lohnenswert erwiesen hat.

Auch wenn die wirtschaftliche Entwicklung in den Neuen Bundesländern abschuissige Tendenz aufweist und damit die Sicherung der Industrieerträge in Form von Aufträgen sich wesentlich schwieriger gestaltet, ist die Arbeitsphilosophie der Fraunhofer Gesellschaft in dieser wirtschaftlich komplizierten Zeit von größter Bedeutung; denn nur das gemeinsame Handeln in Form der Entwicklung echter Innovationen und ihrer sofortigen Umsetzung in unternehmerischen Prozessen wird helfen können, einen kleinen Baustein zur wirtschaftlichen Gesundung beizutragen.

Deshalb sei an dieser Stelle allen Beteiligten am Aufbau und der Unterstiitzung, insbesondere den Herren Prof. Dr.-Ing. Dr. h.c. mult. H.-J. Warnecke als Präsident der Fraunhofer Gesellschaft sowie Herrn Prof. Dr.-Ing. Dr. h.c. mult. Dr.-Ing. E.h. R. Jünemann als Institutsleiter des Fraunhofer Institutes für Materialfluß und Logistik und dem Präsidenten der Technischen Fachhochschule Wildau, Herrn Prof. Dr.-Ing. W. Arlt, herzlich gedankt.

\section{Verfasser}

Prof. Dr.-Ing. habil. Bernd Hentschel

Technische Fachhochschule Wildau

Fachbereich Ingenieurwesen/Wirtschaftsingenieurwesen Fachgebiet: Produktionslogistik

Fraunhofer Anwendungszentrum „Entsorgungs- und Verkehrslogistik“" 